\title{
Peritoneal recurrence in gastric cancer following curative resection can be predicted by postoperative but not preoperative biomarkers: a single-institution study of 320 cases
}

\author{
Fan $\mathbf{W u}^{1}$, Chunmei Shi ${ }^{2,3}$, Riping $\mathbf{W u}^{1,2}$, Zhiqing Huang ${ }^{1,4}$ and Qiang Chen ${ }^{1,4}$ \\ ${ }^{1}$ The Union Clinical Medical College of Fujian Medical University, Fuzhou, Fujian Province, PR, China \\ ${ }^{2}$ Fujian Medical University Union Hospital, Fuzhou, Fujian Province, PR, China \\ ${ }^{3}$ Fujian Key Laboratory of Translational Cancer Medicine, Fuzhou, Fujian Province, PR, China \\ ${ }^{4}$ Fujian Medical University Stem Cell Research Institute, Fuzhou, Fujian Province, PR, China \\ Correspondence to: Qiang Chen, email: cqiang8@189.cn \\ Chunmei Shi, email: scmfz@qq.com
}

Keywords: peritoneal recurrence, gastric cancer, curative resection, postoperative biomarkers

Received: November 01,2016 Accepted: February 28, $2017 \quad$ Published: May 08, 2017

Copyright: Wu et al. This is an open-access article distributed under the terms of the Creative Commons Attribution License 3.0 (CC BY 3.0), which permits unrestricted use, distribution, and reproduction in any medium, provided the original author and source are credited.

\section{ABSTRACT}

To determine the risk factors for peritoneal recurrence in gastric cancer patients after curative resection, we included 320 patients with stage I-III primary gastric cancer between January 2008 and June 2012. Data on each patient's surgical and pathological information, preoperative and postoperative tumor markers were collected and analyzed retrospectively. The risk factors for peritoneal recurrence were investigated by univariate and multivariate analysis. In patients with peritoneal recurrence, advanced $\mathrm{T}$ or $\mathbf{N}$ stage, low differentiation, vascular/lymphatic invasion, perineural invasion, and elevated postoperative CEA/CA19-9 were more common than in patients without peritoneal recurrence. Patients with peritoneal recurrence showed a worse overall survival (OS) compared to those without peritoneal recurrence. In addition, patients with peritoneal recurrence within the first year had a worse OS compared to those with recurrence after 1 year. The univariate and multivariate analyses revealed that elevated number of metastatic lymph nodes and elevated postoperative CEA and CA19-9 were three independent risk factors for peritoneal recurrence in gastric cancer patients. For patients with N3 stage and high postoperative CEA and CA19-9, we found an initial steep slope within approximately 1 year and a subsequent gentle slope in the risk curve. Combined receiver operating characteristic curve analysis using the three independent risk factors for peritoneal recurrence yielded an area under the curve value of 0.73 with $73.7 \%$ sensitivity and $64.2 \%$ specificity. Therefore, the risk factors may be associated with peritoneal recurrence after curative resection in selected gastric cancer patients.

\section{INTRODUCTION}

Gastric cancer is one of the most common cancers and is the second leading cause of cancer-related deaths worldwide. In China, the morbidity and mortality of the disease is more than two fold higher than the world average [1]. Surgery and adjuvant therapy including chemotherapy and radiotherapy are the mainstay of treatment. However, nearly $20 \%$ of gastric cancer patients are diagnosed with peritoneal metastasis before or after surgery, and more than 50\% develop peritoneal recurrence (PR) following curative resection [2]. Moreover, PR can lead to bowel obstruction or malignant ascites, resulting in a poor prognosis and decline in quality of life. Several authors $[3,4]$ have reported that hyperthermic intraperitoneal chemotherapy (HIPEC) or intraperitoneal chemotherapy prevent PR and improve survival among postoperative gastric cancer patients with a high risk of PR. Therefore, it is very important to identify risk factors in order to implement prophylactic measures to prevent 
PR by using additional adjuvant chemotherapies such as HIPEC.

However, early detection of PR remains a challenge, and currently, reliable predictors for PR of gastric cancer are unavailable in clinical practice [5]. Conventional computed tomography (CT) and positron emission tomography (PET) cannot accurately diagnose peritoneal metastasis [6]. Peritoneal lavage cytology (PLC) had been regarded as a reliable method for predicting PR but it is often criticized for having a relatively low sensitivity for detecting free cancer cells and predicting PR [79]. Although the American Joint Committee on Cancer (AJCC) staging system in 2010 included peritoneal cytology in the TNM $7^{\text {th }}$ Edition, with a positive peritoneal cytology staged as M1 disease [10], the sensitivity of PLC is low, with an incidence of positive PLC ranging from $18 \%$ to $35 \%$ [11]. Later studies proved that quantitative RT-PCR of peritoneal washes was more effective and had higher sensitivity than conventional cytological examination as a tool for predicting PR in patients with gastric cancer [12-14]. Recently, Takeno et al. showed that a 22-gene expression profile using oligonucleotide microarrays covering 30,000 human probes gene expression profile from primary gastric cancer tissues can be useful in the prediction of PR after curative surgery [15]. However, these methods had some limitations including strict requirements of sample collection, a complicated and time-consuming operation process, high cost, and false positives.

To date, there is no consensus about which clinicopathological factors or biomarkers can predict PR after curative resection. Furthermore, only a few studies have analyzed the usefulness of postoperative biomarkers in predicting PR. The prognostic effect of clinicopathological factors and postoperative biomarkers such as CEA and CA19-9 remains unclear. In the present study, through an 8-year follow-up, we aim to evaluate the risk factors of $\mathrm{PR}$ in patients with gastric cancer following curative resection, and in particular, to focus on postoperative biomarkers that have not been fully investigated.

\section{MATERIALS AND METHODS}

\section{Patient selection and classification}

The study cohort included 320 patients with nonmetastatic gastric cancer who received curative resection (N2 level) at the Fujian Medical University Union Hospital between January 2008 and June 2012. Patients with prior malignancy were excluded. Data on each patient's gender, age, surgical and pathological information (including resection type, TNM stage, tumor location, differentiation, vascular/lymphatic invasion, perineural invasion [PNI]), and preoperative and postoperative tumor markers (CEA, CA19-9) were collected retrospectively. Postoperative tumor markers were analyzed 4 weeks after surgery to prevent the adjuvant therapy from affecting the outcome.

The primary tumor and regional lymph nodes were classified histologically and staged according to the AJCC TNM staging system based on postoperative pathological reports [16]. Curative resection was defined as complete removal of the primary gastric tumor, D2 resection of regional lymph nodes, and absence of any residual macroscopic tumors. D2 resection (N2 level) was defined as the removal of nodes along the left gastric artery (station 7), common hepatic artery (station 8), celiac trunk (station 9), splenic hilum, and splenic artery (stations 10 and 11). The biomarkers included CEA and CA19-9 levels in peripheral blood samples, which were collected prior to surgery and within one month after surgery.

The primary end-points were disease-free survival (DFS) and overall survival (OS) [17, 18]. DFS was defined as the time from the date of initial surgery to the first event (relapse, metastasis, or death). OS was defined as the period from initial surgery to the time of death. PR was diagnosed when disseminated nodules were found in the peritoneal cavity by imaging studies (CT, abdominal ultrasound [US], MRI, or PET-CT), ascitic cytology, laparoscopy, or laparotomy.

\section{Follow-up}

Patients were generally seen for follow-up within the first month of surgery and then every 3 months after surgery. For patients who discontinued follow-up at our hospital, attempts were made to obtain information via telephonic contact. In total, 21 patients $(21 / 320,6.5 \%)$ were lost to follow-up, and we censored these patients in the analysis. The latest follow-up was at the end of July 2016. The median follow-up duration was 36 months (range: 3-96 months). This retrospective study was approved by the Ethical Committees of Fujian Medical University Union Hospital.

\section{Statistics}

The chi-square test or Fisher's exact test was used to assess the distribution of clinical characteristics between patients with PR and without PR. Survival plots were drawn using the Kaplan-Meier method. Univariate analysis of the differences in prognosis between the two groups was conducted by using the log-rank test. A Cox multivariate regression model was used to identify clinicopathological factors affecting prognosis in the entire cohort. The major covariates were age, gender, type of surgery, histologic type, tumor invasion, lymph node status, tumor location, differentiation, vascular/lymphatic invasion, PNI, adjuvant therapy, and preoperative and 
postoperative biomarkers. Only patients with information for all of the covariates were included in the Cox multivariate regression analyses. Results that had $p$-values $<0.05$ were considered statistically significant. SPSS 19.0 software was used for statistical analysis.

\section{RESULTS}

\section{Clinicopathological characteristics of patients}

During the follow-up period, 188 patients (188/320, $58.8 \%$ ) developed metastasis after curative resection. A total of 77 patients $(77 / 188,41.0 \%)$ developed peritoneal recurrence; other metastatic sites included abdominal wall metastasis in 1 case, lung metastasis in 1 case, pancreas metastasis in 2 cases, bone metastasis in 5 cases, liver metastasis in 10 cases, locoregional (including anastomosis or stump, adjacent organ and regional lymph nodes) recurrence in 15 cases, distant lymph nodes (including retroperitoneal lymph nodes and extraabdominal nodes) in 24 cases, and various combinations of multiple sites.

The clinical characteristics of the 320 patients are shown in Table 1. Gastric cancer with PR was associated with deeper depth of invasion (T stage), more number of metastatic lymph nodes ( $\mathrm{N}$ stage), and advanced TNM stage, and the PR group had more low differentiation, vascular/lymphatic invasion, and PNI. In addition, levels of postoperative biomarkers (elevated CEA and CA19-9) were significantly positively associated with PR in gastric cancer (postoperative CEA, $p=0.005$; postoperative CA19-9, $p=0.001)$. However, age, gender, type of resection, and tumor location were not associated with PM. In addition, elevated pre-operative biomarkers were not associated with peritoneal recurrence.

In the univariate analysis, patients with PR showed a worse OS compared to those without PR, with a median OS of 15 months (95\% confidence interval [CI] 9.7$20.3) v s$. an unreached median OS (log-rank $p<0.001)$, and a hazard ratio [HR] of 7.1 (95\% CI 3.4-14.8) across the entire cohort. At 1 and 3 years after surgery, patients without PR had an OS rate of 86.4\% (95\% CI: 78.2-94.6) and $62.1 \%$ (95\% CI: 50.3-73.9), whereas patients with PR had an OS rate of $60 \%$ (95\% CI: 40.8-79.2) and 16\% (95\% CI: 1.7-30.3), respectively. Survival plots of the two groups are shown in Figure 1.

The median time for PR was 13.3 months. Thirtyseven $(37 / 77,48.0 \%)$ patients developed PR within the first year after surgery, and $26(26 / 77,33.8 \%)$ patients developed PR within 1-2 years after surgery. The remaining patients $(14 / 77,18.2 \%)$ developed PR after 2 years following surgery $(p<0.001)$. Patients who relapsed within the first year had a worse OS than those who developed recurrence beyond 1 year, with a median OS of 12 months vs. 31 months, respectively, $(p<0.001)$ and an HR of 3.89 ( 95\% CI 1.57-9.60). The survival plots of the two groups are shown in Figure 2.

To investigate the prognostic factors of patients with gastric cancer developing PR, we studied many potential clinicopathological factors in univariate and multivariate analysis with a Cox regression model (Table 2 ). The univariate analyses showed that advanced $T$ stage, advanced $\mathrm{N}$ stage, poor differentiation, positive vascular/ lymphatic invasion, positive PNI, elevated preoperative CA19-9, and CEA were associated with developing $\mathrm{PR}$ in gastric cancer patients after curative resection.

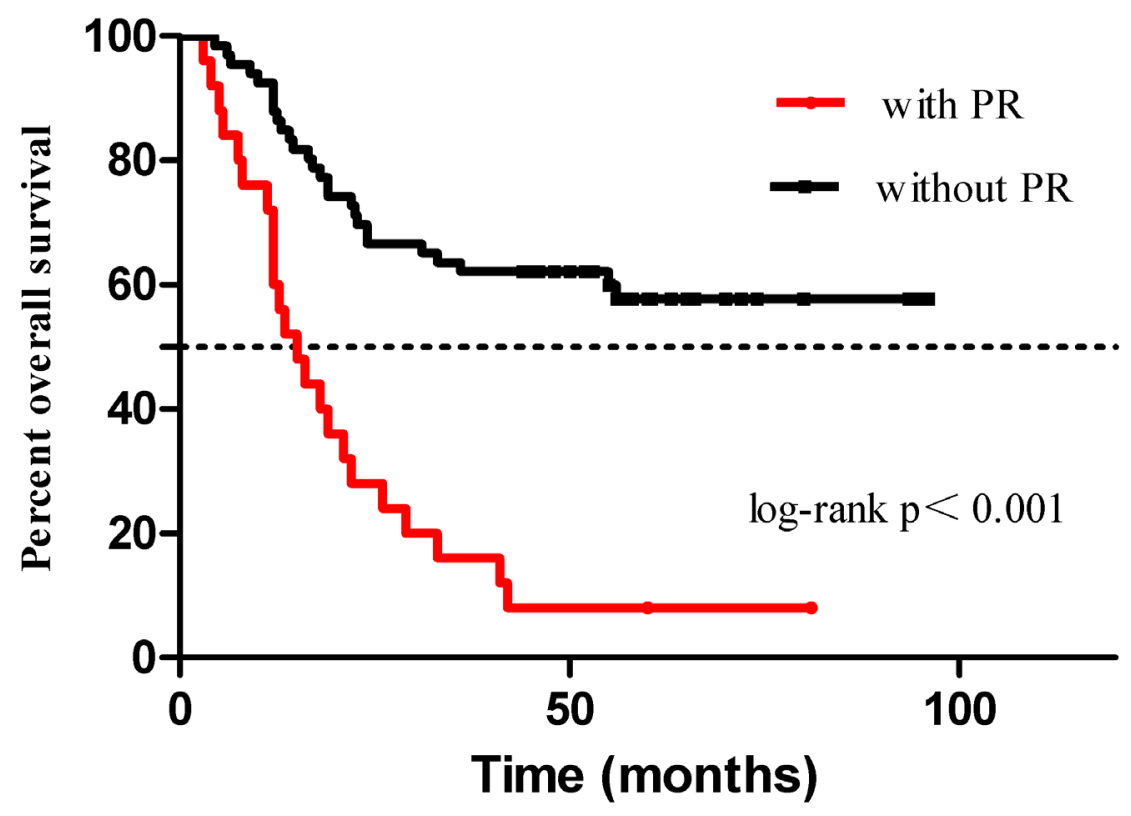

Figure 1: OS of patients with and without PR. 
Table 1: Clinical characteristics of gastric cancer patients with and without PR

\begin{tabular}{|c|c|c|c|}
\hline \multirow[t]{2}{*}{ characteristics } & $\begin{array}{c}\text { Patients with PR } \\
\quad N=77\end{array}$ & $\begin{array}{l}\text { Patients without PR } \\
\quad N=243\end{array}$ & $P$ \\
\hline & $n(\%)$ & $n(\%)$ & \\
\hline Age (years) & & & \multirow{3}{*}{0.62} \\
\hline$<60$ & 36 & 105 & \\
\hline$\geq 60$ & 41 & 138 & \\
\hline \multicolumn{3}{|l|}{ Gender } & \multirow{3}{*}{0.88} \\
\hline male & 57 & 183 & \\
\hline female & 20 & 60 & \\
\hline \multicolumn{3}{|l|}{ Type of resection } & \multirow{3}{*}{0.27} \\
\hline LTG & 56 & 159 & \\
\hline LDG & 21 & 84 & \\
\hline \multicolumn{3}{|l|}{ T stage } & \multirow{4}{*}{$<0.001$} \\
\hline $\mathrm{T} 1 / 2$ & 2 & 53 & \\
\hline $\mathrm{T} 3$ & 26 & 82 & \\
\hline $\mathrm{T} 4$ & 49 & 108 & \\
\hline \multicolumn{3}{|l|}{$\mathrm{N}$ stage } & \multirow{5}{*}{$<0.001$} \\
\hline No & 5 & 55 & \\
\hline N1 & 6 & 31 & \\
\hline $\mathrm{N} 2$ & 13 & 61 & \\
\hline N3 & 53 & 96 & \\
\hline \multicolumn{3}{|l|}{ TNM stage } & \multirow{4}{*}{$<0.001$} \\
\hline I & 1 & 33 & \\
\hline II & 9 & 50 & \\
\hline III & 67 & 160 & \\
\hline \multicolumn{3}{|l|}{ Tumor location } & \multirow{3}{*}{0.07} \\
\hline Proximal & 18 & 84 & \\
\hline Others & 59 & 159 & \\
\hline \multicolumn{3}{|l|}{ Differentiation } & \multirow{3}{*}{0.007} \\
\hline High/moderate & 14 & 84 & \\
\hline Low & 63 & 159 & \\
\hline \multicolumn{3}{|l|}{ Vascular/lymphatic invasion } & \multirow{3}{*}{0.008} \\
\hline Negative & 47 & 187 & \\
\hline Positive & 30 & 56 & \\
\hline \multicolumn{3}{|l|}{ Perineural invasion } & \multirow{3}{*}{0.006} \\
\hline Negative & 59 & 218 & \\
\hline Positive & 18 & 25 & \\
\hline \multicolumn{3}{|l|}{ Preoperative CEA } & \multirow{4}{*}{$\begin{array}{c}0.806 \\
0.891 \text { (high vs. normal) }\end{array}$} \\
\hline Normal & 49 & 160 & \\
\hline High & 22 & 69 & \\
\hline Unknown & 6 & 14 & \\
\hline
\end{tabular}




\begin{tabular}{|c|c|c|c|}
\hline Preoperative & & & \multirow{4}{*}{$\begin{array}{c}0.281 \\
0.180 \text { (high vs. normal) }\end{array}$} \\
\hline Normal & 53 & 189 & \\
\hline High & 19 & 43 & \\
\hline Unknown & 5 & 11 & \\
\hline \multicolumn{3}{|c|}{ Postoperative CEA } & \multirow{4}{*}{$\begin{array}{c}0.019 \\
0.005 \text { (high vs. normal) }\end{array}$} \\
\hline Normal & 50 & 194 & \\
\hline High & 21 & 34 & \\
\hline Unknown & 6 & 15 & \\
\hline \multicolumn{3}{|c|}{ Postoperative CA19-9 } & \multirow{4}{*}{$\begin{array}{c}0.004 \\
0.001 \text { (high vs. normal) }\end{array}$} \\
\hline Normal & 49 & 190 & \\
\hline High & 24 & 35 & \\
\hline Unknown & 4 & 18 & \\
\hline \multicolumn{4}{|c|}{ adjuvant therapy } \\
\hline yes & 66 & 193 & 0.248 \\
\hline no & 11 & 50 & \\
\hline
\end{tabular}

LTG: laparoscopic total gastrectomy, LDG: laparoscopic distal gastrectomy

The prognostic indicators identified in the univariate analyses were further studied in multivariate analyses. Cox regression analysis indicated that advanced $\mathrm{N}$ stage and elevated postoperative CEA and CA19-9 were three independent risk factors for PR. Furthermore, patients with 1-6 metastatic lymph nodes did not have a higher risk of PR than patients with no lymph node metastasis $(p$ $=0.239$ ), but patients with more than 7 metastatic lymph nodes showed a higher risk of PR than patients with no lymph node metastasis, with a HR of 9.43 (95\% CI 2.2439.70) $(p=0.002)$. In addition, elevated postoperative CEA and CA19-9 were prognostic factors for PR with HRs of 2.93 (95\% CI 1.42-6.06) and 3.58 (95\% CI 1.63$7.87)$, respectively. The detailed findings are presented in Table 2.

To further evaluate the priority of these three risk factors for predicting PR, analysis was performed in recurrent patients but not whole patient group. And we

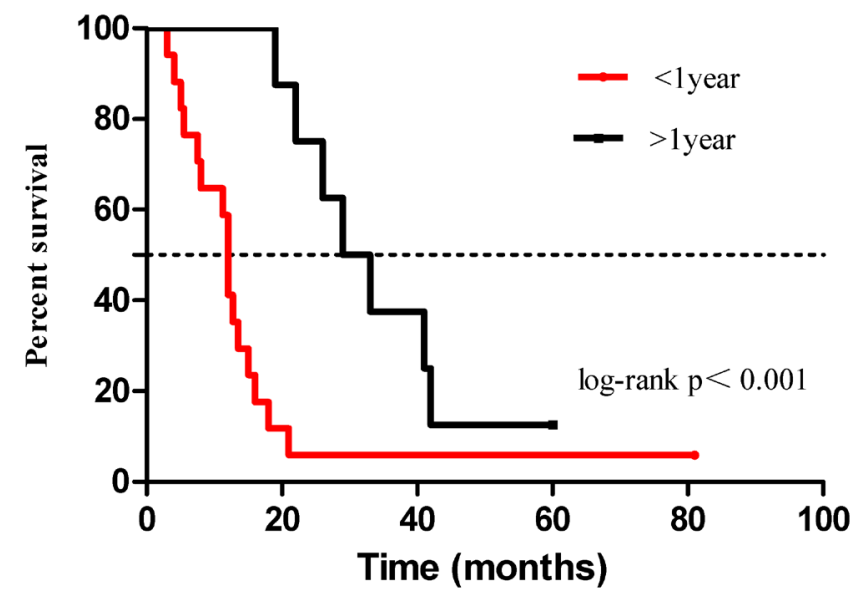

Figure 2: OS of patients with early recurrence and late recurrence. The median overall survival time of patients with early recurrence (within 1 year) was significantly shorter than that of patients with late recurrence (after 1 year) (12 vs. 31 months, respectively, $p<0.001$ ) for gastric cancer patients with PR. 
Table 2: Univariate and multivariate analysis of risk factors for PR

\begin{tabular}{|c|c|c|c|c|c|c|}
\hline \multirow{2}{*}{ Factors } & \multicolumn{3}{|c|}{ Univariate analysis } & \multicolumn{3}{|c|}{ Multivariate analysis* } \\
\hline & HR & $95 \% \mathrm{CI}$ & $p$ & HR & $95 \% \mathrm{CI}$ & $p$ \\
\hline Age $(<60 v s . \geq 60)$ & 1.01 & $0.99-1.03$ & 0.37 & - & - & - \\
\hline Gender (Male vs. Female) & 1.00 & $0.60-1.66$ & 0.987 & - & - & - \\
\hline Type of resection (LTG $v s$. LDG) & 0.68 & $0.41-1.13$ & 0.138 & - & - & - \\
\hline T stage (T3/4 vs. T1/2) & 2.40 & $1.50-3.82$ & $<0.001$ & 2.60 & $0.26-25.78$ & 0.414 \\
\hline $\mathrm{N}$ stage (N3 vs. N1/2 vs. N0) & & & $<0.001$ & & & $<0.001$ \\
\hline N1/2 vs. N0 & 2.75 & $1.09-6.89$ & 0.031 & 2.26 & $0.25-20.47$ & 0.467 \\
\hline N3 vs. N0 & 8.68 & $3.69-20.41$ & $<0.001$ & 11.46 & $1.10-119.90$ & 0.042 \\
\hline TNM stage (I vs. II vs. III ) & & & $<0.001$ & - & - & - \\
\hline II $v s . I$ & 4.28 & $1.23-14.88$ & 0.022 & - & - & - \\
\hline III vs.II & 3.20 & $2.26-4.52$ & $<0.001$ & - & - & - \\
\hline III $v s . \mathrm{I}$ & 3.85 & $2.64-5.60$ & $<0.001$ & - & - & - \\
\hline Location (Proximal vs. distal) & 1.20 & $0.71-2.04$ & 0.491 & - & - & - \\
\hline Differentiation (poor vs. well) & 2.96 & $1.59-5.50$ & 0.001 & 1.02 & $0.46-2.28$ & 0.961 \\
\hline Vascular/lymphatic invasion $(+v s .-)$ & 2.23 & $1.41-3.54$ & 0.001 & 1.26 & $0.63-2.44$ & 0.544 \\
\hline Perineural invasion $(+v s .-)$ & 1.72 & $1.00-2.95$ & 0.049 & 1.43 & $0.78-2.63$ & 0.248 \\
\hline Preoperative CEA (high vs. normal) & 1.24 & $0.68-2.26$ & 0.49 & - & - & - \\
\hline Preoperative CA199 (high vs. normal) & 1.87 & $1.01-3.45$ & 0.045 & 1.14 & $0.58-2.34$ & 0.697 \\
\hline Postoperative CEA (high vs. normal) & 3.62 & $1.79-7.34$ & $<0.001$ & 3.13 & 1.54-6.34 & 0.002 \\
\hline Postoperative CA199 (high vs. normal) & 4.73 & $2.20-10.20$ & $<0.001$ & 3.97 & $1.78-8.84$ & 0.001 \\
\hline
\end{tabular}

*The multivariate analysis was stratified by TNM stage because there was a positive association between high preoperative/ postoperative biomarkers ( CEA and CA19-9) and tumor stage with low strengths (data not shown)

found that elevated number of metastatic lymph nodes and elevated postoperative CEA/CA19-9 were still three independent risk factors for PR within recurrent patients (Table 3)

Kaplan-Meier survival curves also revealed that an increased number of metastatic lymph node was associated with an increased rate of PR $(p<0.001)$ (Figure 3 ). Patients with stage N0, N1-2, and N3 had a 1-year and 2-year risk of PR of $0 \%, 10.85 \%$, and $25.69 \%(p<0.001)$ and $5.26 \%, 22.03 \%$, and $47.46 \%(p<0.001)$, respectively. Patients with N3 stage had a higher recurrence risk compared to those with N1-2 $(p<0.001)$ and N0 $(p<$ $0.001)$ stages, respectively, and patients with N1-2 stage also had a higher recurrence risk compared to those with N0 $(p=0.017)$.

Elevated postoperative CEA and CA19-9 were associated with a higher risk of PR compared to normal postoperative CEA $(p<0.001)$ (Figure 4). and CA19-9

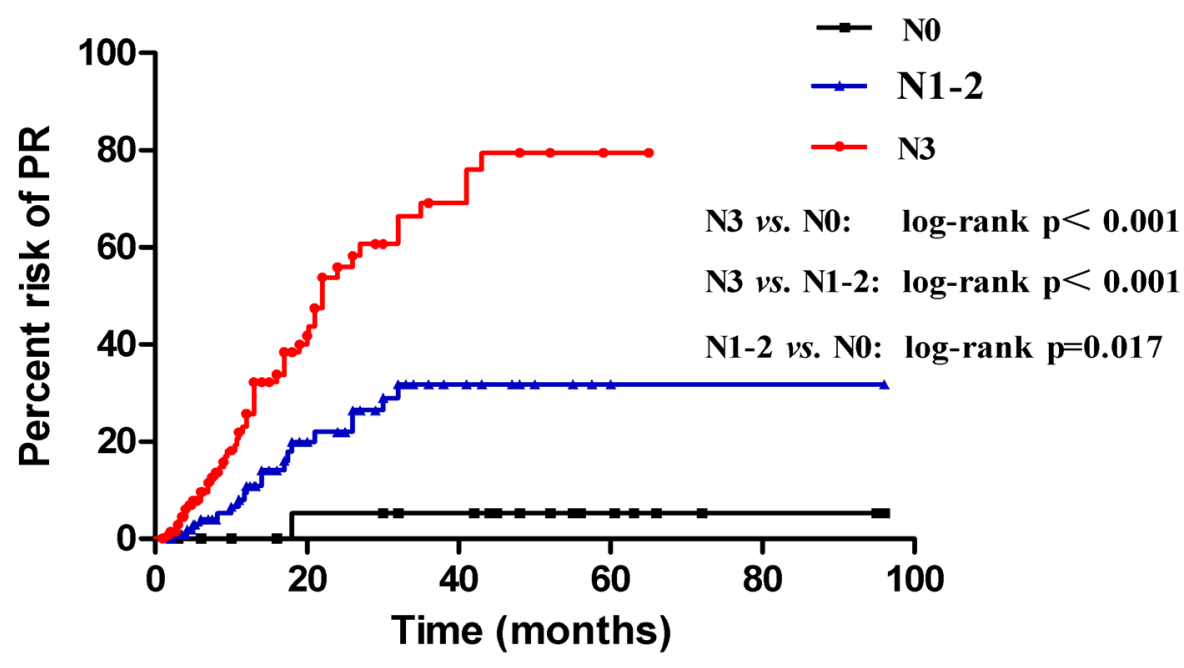

Figure 3: Risk of PR between three subgroups: N0, N1-2, and N3. 
Table 3: Univariate and multivariate analysis of risk factors for PR in recurrent patients

\begin{tabular}{|c|c|c|c|c|c|c|}
\hline \multirow{2}{*}{ Factors } & \multicolumn{3}{|c|}{ Univariate analysis } & \multicolumn{3}{|c|}{ Multivariate analysis* } \\
\hline & HR & $95 \% \mathrm{CI}$ & $p$ & HR & $95 \% \mathrm{CI}$ & $p$ \\
\hline Age $(<60 v s . \geq 60)$ & 1.13 & $0.72-1.80$ & 0.593 & - & - & - \\
\hline Gender (Male $v s$. Female) & 0.82 & $0.48-1.40$ & 0.427 & - & - & - \\
\hline Type of resection (LTG $v s$. LDG) & 0.68 & $0.41-1.13$ & 0.095 & - & - & - \\
\hline T stage (T4 vs. T2/3) & 1.04 & $0.65-1.64$ & 0.881 & 1.39 & $0.72-2.68$ & 0.332 \\
\hline $\mathrm{N}$ stage (N3 vs. N1/2) & 1.68 & $1.07-2.61$ & 0.029 & 2.39 & $1.10-5.17$ & 0.027 \\
\hline TNM stage (I vs. II vs. III ) & & & 0.887 & - & - & - \\
\hline II $v s$. I & 1.18 & $0.17-8.19$ & 0.868 & - & - & - \\
\hline III $v s$. II & 1.19 & $0.61-2.27$ & 0.636 & - & - & - \\
\hline III $v s$. I & 1.14 & $0.18-7.27$ & 0.898 & - & - & - \\
\hline Location (Proximal vs. distal) & 1.25 & $0.76-2.07$ & 0.391 & - & - & - \\
\hline Differentiation (poor $v s$. well) & 1.51 & $0.89-2.56$ & 0.125 & 1.20 & $0.55-2.60$ & 0.653 \\
\hline Vascular/lymphatic invasion (+ vs. - ) & 1.81 & $1.09-3.02$ & 0.008 & 1.71 & $0.93-3.15$ & 0.086 \\
\hline Perineural invasion $(+v s .-)$ & 1.20 & $0.69-2.09$ & 0.497 & - & - & - \\
\hline Preoperative CEA (high vs. normal) & 1.23 & $0.71-2.14$ & 0.425 & - & - & - \\
\hline Preoperative CA199 (high vs. normal) & 1.63 & $1.01-3.43$ & 0.011 & 1.14 & $0.43-1.78$ & 0.703 \\
\hline Postoperative CEA (high $v s$. normal) & 2.55 & $1.11-5.85$ & 0.027 & 2.49 & $1.21-5.10$ & 0.013 \\
\hline Postoperative CA199 (high vs. normal) & 3.53 & $1.08-11.60$ & $<0.001$ & 3.45 & $1.48-8.08$ & 0.004 \\
\hline
\end{tabular}

*The multivariate analysis was stratified by TNM stage because there was a positive association between high preoperative/ postoperative biomarkers ( CEA and CA19-9) and tumor stage with low strengths (data not shown)

$(p<0.001)$ (Figure 5). In addition, normal postoperative CEA had a 1-year and 2-year risk of PR of $13.37 \%$, $30.46 \%$, whereas elevated postoperative CEA had a 1-year and 2-year risk of PR of 33.88\% $(p<0.001)$ and $64.74 \%$ $(p<0.001)$, respectively. Normal postoperative CA19-9 had a 1-year and 2-year risk of PR of 12.81\%, 32.25\%, whereas high postoperative CA19-9 had a 1-year and 2-year risk of PR of $53.78 \%(p<0.001)$ and $69.19 \%(p<$ $0.001)$, respectively. For patients with N3 stage and high postoperative CEA and CA19-9, we found an initial steep slope within approximately 1 year and a subsequent gentle slope in the risk curve.

The number of metastatic lymph nodes between patients with and without PR evaluated by a scatter diagram(Figure 6). PR patients had a median metastatic lymph node number of 13 compared to 5 in patients without PR $(p<0.001)$

Combined receiver operating characteristic (ROC) curve analysis using the three independent risk factors, including number of metastatic lymph nodes,

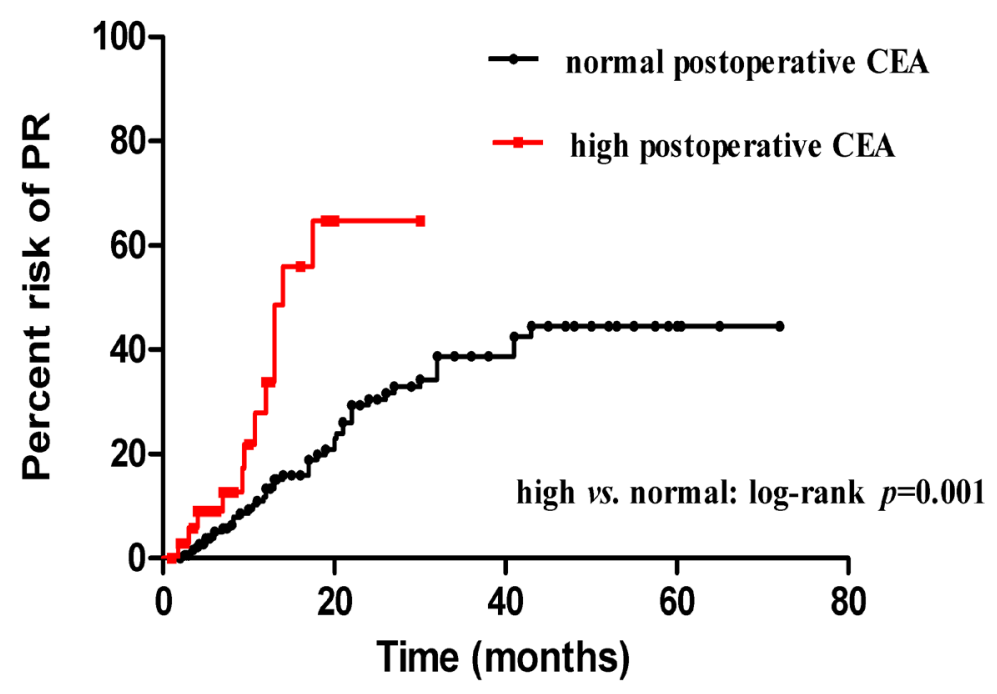

Figure 4: Risk of PR between two subgroups: elevated and normal postoperative CEA. 


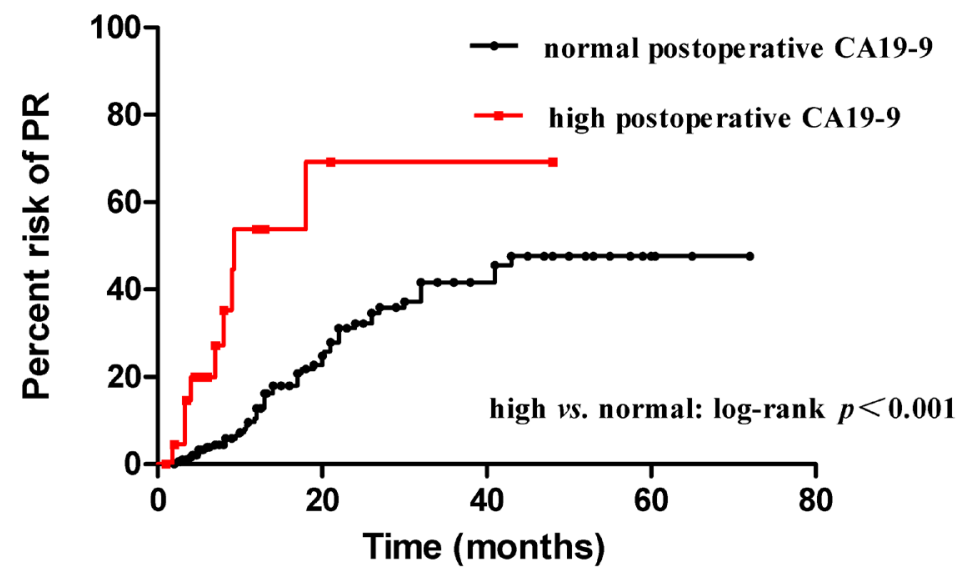

Figure 5: Risk of PR between two subgroups: elevated and normal postoperative CA19-9.

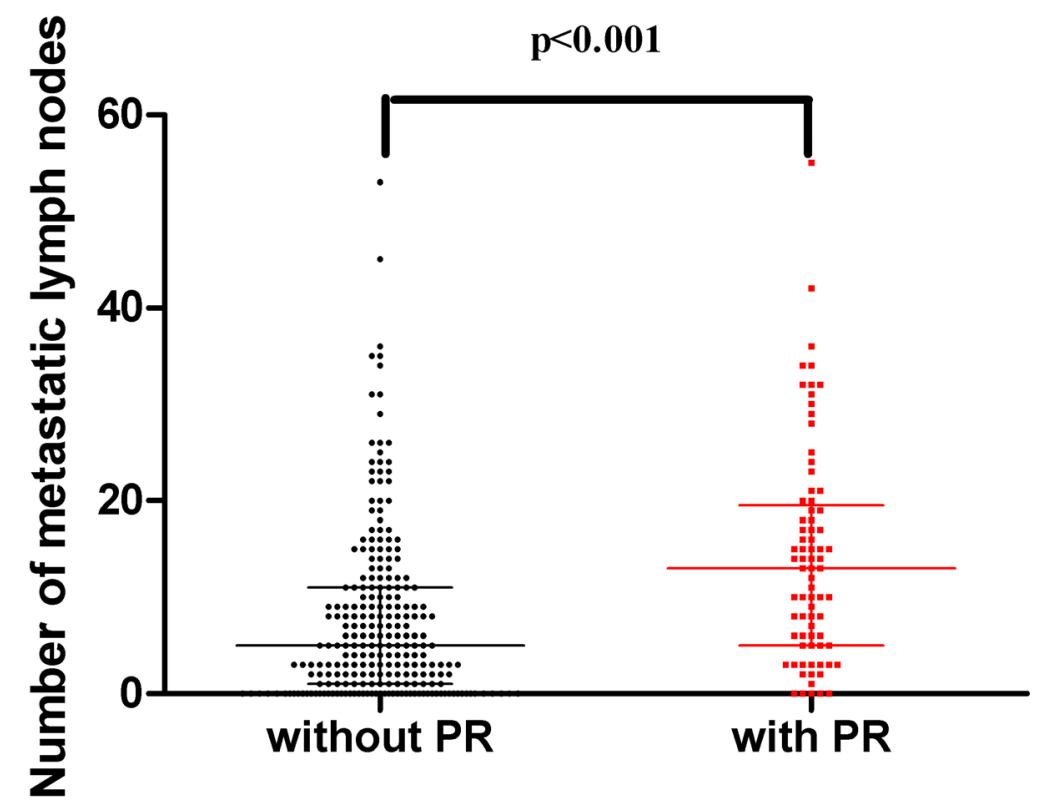

Figure 6: Number of metastatic lymph nodes between patients with and without PR.

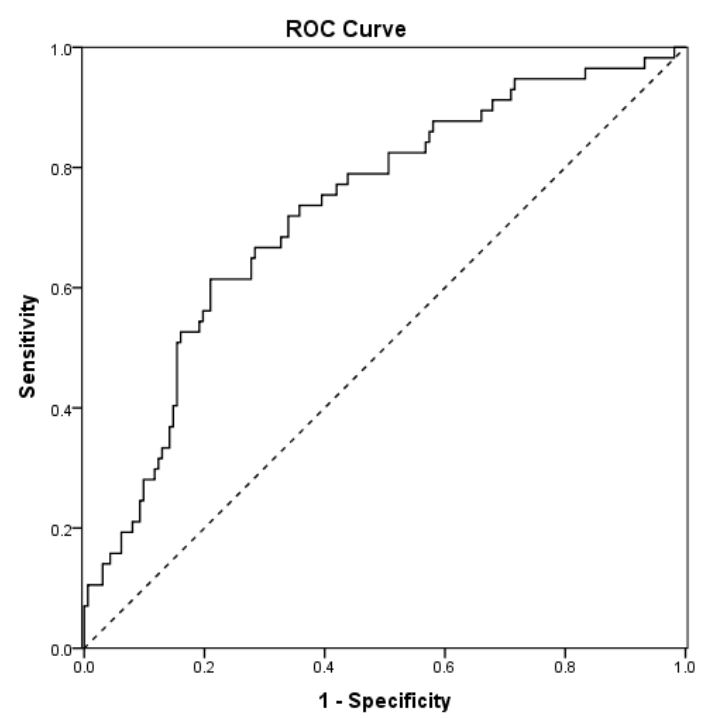

Figure 7: Combined ROC curve analysis using the three independent risk factors for predicting PR. 
postoperative CEA, and postoperative CA19-9 levels for predicting $\mathrm{PR}$ in gastric cancer patients yielded an area under the curve (AUC) value of 0.73 (95\% CI 0.65-0.81) with $73.7 \%$ sensitivity and $64.2 \%$ specificity (Figure 7).

\section{DISCUSSION}

The development of PR in patients with gastric cancer is associated with a poor prognosis, and adversely affects the quality of life [19]. Randomized trials of adjuvant intraperitoneal chemotherapy revealed a significant reduction of PR in gastric cancer patients [3, 20]. However, there are some side effects such as chemical peritonitis, pain, intestinal adhesion, etc. Therefore, it is important to find risk factors related to PR and determine a suitable population for intraperitoneal perfusion chemotherapy in patients with stage I-III gastric cancer after curative resection.

At present, it remains unclear which clinical factors can predict PR in gastric cancer patients after surgery. US, CT and PET/CT are commonly used for detecting PR [21-23]. However, a meta-analysis revealed that US and CT did not have a consistently high sensitivity and specificity in assessing PR in gastric cancer patients [24]. In addition, the 18F-FDG method for detecting PR is too expensive, although it has high sensitivity and specificity. [25]. Recent developments in staging laparoscopy have helped overcome these problems and has become the main tool to predict PR in gastric cancer patients [26, 27]. However, there is no clear indication for the use of staging laparoscopy to detect PR in gastric cancer patients, and it is an invasive procedure that cannot be routinely performed during follow-up. Therefore, by analyzing long-term outcomes, our study focused on the prognostic factors that could predict PR after curative resection in stage I-III gastric cancer patients.

In the present study, we found that PR was the most frequent pattern of metastasis after curative resection, with a frequency of occurrence of approximately $40 \%$, which is consistent with previous studies [28-30]. Previous studies had shown that advanced TNM stage, differentiation, and venous/lymphatic invasion were more frequent in patients with PR [31,32]. We also found that PR was associated with advanced $\mathrm{T}$ stage, advanced $\mathrm{N}$ stage, low differentiation grade of the primary tumor, and vascular/lymphatic invasion. In addition, a previous study showed that PNI positivity in gastric cancer patients after curative resection was associated with poor prognosis [33]. Furthermore, our data revealed that PNI positivity was found more frequently in patients with PR than those without PR, which was reported in only in a few previous studies. In addition, higher levels of postoperative biomarkers (CEA and CA19-9) were significantly and positively associated with $\mathrm{PR}$ in gastric cancer patients (postoperative CEA: $p=0.001$; postoperative CA19-9: $p$
$=0.007)$. These findings are consistent with those of other studies [31,34]. However, one study did not find any such correlation between CA 19-9 and CEA, and PR [35]; these differences might be due to the $\mathrm{D} 1$ lymph node dissection carried out in that study, and its relatively small sample size.

Our results showed that patients with PR had a worse OS (median OS: 15 months) than those without PR (vs unreached median OS) using univariate analysis (log-rank $p<0.001$; HR, 7.1; 95\% CI, 3.4-14.8). Fukuchi et al. also found that patients with PR had a median OS between 14 and 17 months [36]. Results from the EVOCAPE 1, a multicentric prospective study [37], revealed that gastric cancer patients with PR had a median OS of only 6.5 months. However, more than half (73/125, $58.4 \%$ ) of these patients had peritoneal carcinomatosis at the time of primary gastric cancer diagnosis, which decreased the survival rates. In addition, advances in adjuvant chemotherapy and chemohyperthermic peritoneal perfusion also improved the OS of patients with gastric cancer.

In the present study, the median time for PR was 13.3 months, which was also found in another study [38]. Our data also suggested that more than $80 \%$ of patients developed PR within 2 years of curative resection, especially within 1 year with a rate of $48.0 \%$. Furthermore, patients who developed PR within 1 year had a worse prognosis than those who developed PR after 1 year, with a HR of 3.89 (95\% CI, 1.57-9.60). Therefore, this finding indicated that more attention should be focused on PR within the first year after surgery during follow-up for those patients with high risk of PR.

To determine the independent risk factors for predicting PR in gastric cancer patients following curative resection, we performed univariate and multivariate analyses while adjusting for multiple factors. The univariate analyses showed that deeper depth of tumor invasion ( $\mathrm{T}$ stage), more number of metastatic lymph nodes (N stage), poor differentiation, positive vascular/ lymphatic invasion, positive PNI, elevated preoperative CA19-9, and elevated postoperative CEA and CA199 predicted PR in gastric cancer patients after curative resection. Multivariate analyses by Cox regression indicated that $\mathrm{N} 3$ stage and elevated postoperative CEA and CA19-9 were the three independent risk factors for predicting PR.

There have been several studies investigating the risk factors for predicting PR in gastric cancer patients, but with varied results $[36,39,40]$. Most studies found advanced $\mathrm{T}$ and $\mathrm{N}$ stage, and poor differentiation were risk factors for predicting PR; these results were also found in the present study. However, the role of serum biomarkers, like CEA or CA19-9, in predicting PR in gastric cancer patients was much more controversial. Masaki Ohi et al. indicated that preoperative CA19-9 was an independent risk factor for predicting PR [31], but other studies [35, 
41] have shown different results. Studies of postoperative biomarkers [42, 43] have shown that postoperative normalized CEA or CA19-9 levels can be used as good prognostic factors in patients who undergo curative gastric resection.

However, there is no evidence on the use of postoperative serum biomarkers as predictors of PR in gastric cancer patients. Our data showed that postoperative but not preoperative biomarkers could predict PR in gastric cancer patients after curative resection. Therefore, the patients with N3 stage (more than 7 metastatic lymph nodes), elevated postoperative CEA and CA19-9 as a high-risk group for PR; following further randomized prospective studies, patients with these risk factors should be considered for receiving adjuvant HIPEC treatment.

Other studies have indicated that intraoperative lavage cytology could predict PR, but this remains controversial due to its low sensitivity [7, 44, 45]. In addition, evaluating the CEA level in peritoneal lavage fluid (pCEA) [46] or evaluating pCEA expression by RTPCR $[12,13]$ were considered to be more suitable than intraoperative lavage cytology in predicting PR. However, these results also remain controversial [47]; additionally, obtaining peritoneal wash fluid is time consuming and inconvenient. Therefore, further research is needed to find predictors of PR by combining risk factors including postoperative serum biomarkers, pCEA expression by RTPCR and other clinical pathological factors. We believe this approach would be more suitable for predicting PR [48].

To further elucidate the role of postoperative CEA, CA19-9, and metastatic lymph nodes in predicting PR, we used the Kaplan-Meier method to compare the risk of PR between different numbers of metastatic lymph nodes. Our results showed that advanced $\mathrm{N}$ stage was positively associated with an increased risk of PR ( $p<$ 0.001). Univariate analysis showed that patients with more than 7 metastatic lymph nodes (N3) had a higher risk of PR than those with N1-2 $(p<0.001)$ or N0 ( $p<$ $0.001)$; patients with N1-2 also had a higher recurrence risk compared to those with N0 $(p=0.017)$. These results also proved that $\mathrm{N} 3$ stage was an independent predictor of PR in patients with gastric cancer, which was also revealed by multivariate analysis. We further compared the number of metastatic lymph nodes between patients with and without PR by a scatter diagram. PR patients had a median metastatic lymph nodes number of 13 compared to 5 in patients without PR $(p<0.001)$, which suggested that the number of metastatic lymph nodes could predict PR, especially in patients with N3 stage. We also compared the risk of PR between elevated postoperative CEA and CA19-9 and normal postoperative CEA and CA19-9 by the Kaplan-Meier method. Elevated postoperative CEA and CA19-9 had a higher risk of PR compared to normal postoperative CEA and CA199, with an initial steep slope within the first year and a subsequent gentle slope in the risk curve. The results were consistent with those of other studies [42, 43]. An advantage of our study is the time of evaluation of postoperative biomarkers, which avoided the bias of adjuvant chemotherapy or chemohyperthermic peritoneal perfusion.

Next, we performed ROC curve analysis using independent clinical predictors including the number of metastatic lymph nodes, elevated postoperative CEA, and elevated postoperative CA19-9 for predicting PR in gastric cancer patients. This yielded an AUC value of 0.73 (95\% CI, 0.65-0.81) with 73.7\% sensitivity and 64.2\% specificity for predicting PR. These findings suggested that these three independent factors could predict PR after curative resection. Although future studies with a larger cohort and longer follow-up are needed, our results might be useful in counseling gastric patients after curative resection about treatment options including adjuvant chemotherapy or chemohyperthermic peritoneal perfusion.

There are several limitations to this study. First, it is a retrospective study and must be interpreted with caution because of the increased likelihood of false-positives and false-negatives. Second, the small sample size of this study, which widens the CIs of the outcomes, is not enough to obtain a convincing result. Last, the information of PLC and the CEA level in peritoneal lavage fluid (pCEA) or pCEA expression by RT-PCR were not evaluated in the present study. Further study would be able to produce more convincing results and have greater implications for clinical practice when combined with information of PLC or pCEA.

In summary, this study suggests that patients with PR had a worse OS when compared to patients without PR, and patients who developed PR within the first year had a worse prognosis than those who relapsed after 1 year. Furthermore, elevated postoperative but not preoperative CEA and CA19-9 could predict peritoneal recurrence, and N3 stage was also an important independent predictor for PR in gastric cancer patients following curative resection. And we found an initial steep slope within approximately 1 year and a subsequent gentle slope in the risk curve for those patients with N3 stage and high postoperative CEA and CA19-9. Combined ROC curve analysis using these three independent clinical predictors could predict PR in gastric cancer patients. These findings might be helpful in selecting patients who would be eligible to receive adjuvant HIPEC. Randomized prospective studies are needed to further validate these clinical predictors.

\section{ACKNOWLEDGMENTS}

The project was supported by the Critical Patented Project of The Science \& Technology Bureau of Fujian Province, Peoples Republic of China (Grant No. 2013YZ0002-2), Special Program for the Development of Strategic Emerging Industries of Fujian Province, Peoples Republic of China (2060404). 


\section{CONFLICTS OF INTEREST}

The authors declared that they have no conflicts of interest in this work.

\section{REFERENCES}

1. Chen W, Zheng R, Baade PD, Zhang S, Zeng H, Bray F, Jemal A, Yu XQ, He J. Cancer statistics in China, 2015. CA: a cancer journal for clinicians. 2016; 66:115-132.

2. Kikuchi H, Kamiya K, Hiramatsu Y, Miyazaki S, Yamamoto M, Ohta M, Baba S, Konno H. Laparoscopic narrow-band imaging for the diagnosis of peritoneal metastasis in gastric cancer. Annals of surgical oncology. 2014; 21:3954-3962.

3. Yonemura Y, de Aretxabala X, Fujimura T, Fushida S, Katayama K, Bandou E, Sugiyama K, Kawamura T, Kinoshita K, Endou Y, Sasaki T. Intraoperative chemohyperthermic peritoneal perfusion as an adjuvant to gastric cancer: final results of a randomized controlled study. Hepato-gastroenterology. 2001; 48:1776-1782.

4. Kuramoto M, Shimada S, Ikeshima S, Matsuo A, Yagi Y, Matsuda M, Yonemura Y, Baba H. Extensive intraoperative peritoneal lavage as a standard prophylactic strategy for peritoneal recurrence in patients with gastric carcinoma. Annals of surgery. 2009; 250:242-246.

5. Yamamoto M, Rashid OM, Wong J. Surgical management of gastric cancer: the East vs. West perspective. Journal of gastrointestinal oncology. 2015; 6:79-88.

6. Altini C, Niccoli Asabella A, Di Palo A, Fanelli M, Ferrari C, Moschetta M, Rubini G. 18F-FDG PET/CT role in staging of gastric carcinomas: comparison with conventional contrast enhancement computed tomography. Medicine. 2015; 94:e864.

7. Bando E, Yonemura Y, Takeshita Y, Taniguchi K, Yasui T, Yoshimitsu Y, Fushida S, Fujimura T, Nishimura G, Miwa K. Intraoperative lavage for cytological examination in 1,297 patients with gastric carcinoma. American journal of surgery. 1999; 178:256-262.

8. Bentrem D, Wilton A, Mazumdar M, Brennan M, Coit D. The value of peritoneal cytology as a preoperative predictor in patients with gastric carcinoma undergoing a curative resection. Annals of surgical oncology. 2005; 12:347-353.

9. Fukagawa T, Katai H, Saka M, Morita S, Sasajima Y, Taniguchi H, Sano T, Sasako M. Significance of lavage cytology in advanced gastric cancer patients. World journal of surgery. 2010; 34:563-568.

10. Chae S, Lee A, Lee JH. The effectiveness of the new (7th) UICC N classification in the prognosis evaluation of gastric cancer patients: a comparative study between the 5 th $/ 6$ th and 7th UICC N classification. Gastric cancer. 2011; 14:166-171.

11. Kang Y, Li S, Ge Q, Liu X, Yang Z, Xue Y, Wang F. Extent of Serosal Changes Predicts Peritoneal Recurrence and
Poor Prognosis After Curative Surgery for Gastric Cancer. Medicine. 2015; 94:e1750.

12. Chae HD, Kim IH. Prognostic significance of CEA expression by RT-PCR in peritoneal wash from patients with gastric cancer: result of a 5-year followup after curative resection. Scandinavian journal of gastroenterology. 2016; 51:956-960.

13. Kodera Y, Nakanishi H, Ito S, Yamamura Y, Kanemitsu Y, Shimizu Y, Hirai T, Yasui K, Kato T, Tatematsu M. Quantitative detection of disseminated free cancer cells in peritoneal washes with real-time reverse transcriptasepolymerase chain reaction: a sensitive predictor of outcome for patients with gastric carcinoma. Annals of surgery. 2002; 235:499-506.

14. Kodera Y, Nakanishi H, Ito S, Yamamura Y, Kanemitsu Y, Shimizu Y, Hirai T, Yasui K, Kato T, Tatematsu M. Quantitative detection of disseminated cancer cells in the greater omentum of gastric carcinoma patients with real-time RT-PCR: a comparison with peritoneal lavage cytology. Gastric cancer. 2002; 5:69-76.

15. Takeno A, Takemasa I, Seno S, Yamasaki M, Motoori M, Miyata H, Nakajima K, Takiguchi S, Fujiwara Y, Nishida T, Okayama T, Matsubara K, Takenaka Y, et al. Gene expression profile prospectively predicts peritoneal relapse after curative surgery of gastric cancer. Annals of surgical oncology. 2010; 17:1033-1042.

16. Edge SB, Byrd DR, Compton CC, Fritz AG, Greene FL, Trotti A. AJCC (American Joint Committee on Cancer) Cancer Staging Manual. 7th ed. New York (NY): Springer; 2010. p. 117.

17. Mauriac L, Pippen JE, Quaresma Albano J, Gertler SZ, Osborne CK. Fulvestrant (Faslodex ${ }^{\mathrm{TM}}$ ) versus anastrozole for the second-line treatment of advanced breast cancer in subgroups of postmenopausal women with visceral and nonvisceral metastases: combined results from two multicentre trials. European journal of cancer. 2003; 39:1228-1233.

18. Cardoso F, Costa A, Norton L, Senkus E, Aapro M, Andre F, Barrios CH, Bergh J, Biganzoli L, Blackwell KL, Cardoso MJ, Cufer T, El Saghir N, et al. ESO-ESMO 2nd international consensus guidelines for advanced breast cancer (ABC2). Breast. 2014; 23:489-502.

19. Chau I, Norman AR, Cunningham D, Waters JS, Oates J, Ross PJ. Multivariate prognostic factor analysis in locally advanced and metastatic esophago-gastric cancer-pooled analysis from three multicenter, randomized, controlled trials using individual patient data. Journal of clinical oncology. 2004; 22:2395-2403.

20. Yu W, Whang I, Chung HY, Averbach A, Sugarbaker $\mathrm{PH}$. Indications for early postoperative intraperitoneal chemotherapy of advanced gastric cancer: results of a prospective randomized trial. World journal of surgery. 2001; 25:985-990.

21. Seevaratnam R, Cardoso R, McGregor C, Lourenco L, Mahar A, Sutradhar R, Law C, Paszat L, Coburn N. How useful is preoperative imaging for tumor, node, metastasis 
(TNM) staging of gastric cancer? A meta-analysis. Gastric cancer. 2012; 15 Suppl 1:S3-18.

22. Cidon EU and Cuenca IJ. Gastric Adenocarcinoma: Is Computed Tomography (CT) Useful in Preoperative Staging? Clinical medicine Oncology. 2009; 3:91-97.

23. Hallinan JT, Venkatesh SK. Gastric carcinoma: imaging diagnosis, staging and assessment of treatment response. Cancer imaging. 2013; 13:212-227.

24. Wang Z, Chen JQ. Imaging in assessing hepatic and peritoneal metastases of gastric cancer: a systematic review. BMC gastroenterology. 2011; 11:19.

25. Ozkan E, Araz M, Soydal C, Kucuk ON. The role of $18 \mathrm{~F}-\mathrm{FDG}-\mathrm{PET} / \mathrm{CT}$ in the preoperative staging and posttherapy follow up of gastric cancer: comparison with spiral CT. World journal of surgical oncology. 2011; 9:75.

26. Tourani SS, Cabalag C, Link E, Chan ST, Duong CP. Laparoscopy and peritoneal cytology: important prognostic tools to guide treatment selection in gastric adenocarcinoma. ANZ journal of surgery. 2015; 85:69-73.

27. De Andrade JP, Mezhir JJ. The critical role of peritoneal cytology in the staging of gastric cancer: an evidence-based review. J Surg Oncol. 2014; 110:291-297.

28. Sasako M, Sano T, Yamamoto S, Kurokawa Y, Nashimoto A, Kurita A, Hiratsuka M, Tsujinaka T, Kinoshita T, Arai K, Yamamura Y, Okajima K. D2 lymphadenectomy alone or with para-aortic nodal dissection for gastric cancer. N Engl J Med. 2008; 359:453-462.

29. Kawamura Y, Satoh S, Umeki Y, Ishida Y, Suda K, Uyama I. Evaluation of the recurrence pattern of gastric cancer after laparoscopic gastrectomy with D2 lymphadenectomy. SpringerPlus. 2016; 5:821.

30. D'Angelica M, Gonen M, Brennan MF, Turnbull AD, Bains M, Karpeh MS. Patterns of initial recurrence in completely resected gastric adenocarcinoma. Annals of surgery. 2004; 240:808-816.

31. Ohi M, Mori K, Toiyama Y, Mohri Y, Okigami M, Yasuda H, Saigusa S, Tanaka K, Inoue Y, Kusunoki M. Preoperative prediction of peritoneal metastasis in gastric cancer as an indicator for neoadjuvant treatment. Anticancer research. 2015; 35:3511-3518.

32. Guo L, Ou JL, Zhang T, Ma L, Qu LF. Effect of expressions of tumor necrosis factor alpha and interleukin $1 \mathrm{~B}$ on peritoneal metastasis of gastric cancer. Tumour biology. 2015; 36:8853-8860.

33. Bilici A, Seker M, Ustaalioglu BB, Kefeli U, Yildirim E, Yavuzer D, Aydin FM, Salepci T, Oncel M, Gumus M. Prognostic significance of perineural invasion in patients with gastric cancer who underwent curative resection. Annals of surgical oncology. 2010; 17:2037-2044.

34. Hwang GI, Yoo CH, Sohn BH, Shin JH, Park YL, Kim HD, Kim YS, Han WK, Pae WK. Predictive value of preoperative serum CEA, CA19-9 and CA125 levels for peritoneal metastasis in patients with gastric carcinoma. Cancer research and treatment. 2004; 36:178-181.
35. Duraker N, Celik AN. The prognostic significance of preoperative serum CA 19-9 in patients with resectable gastric carcinoma: comparison with CEA. J Surg Oncol. 2001; 76:266-271.

36. Fukuchi M, Mochiki E, Ishiguro T, Ogura T, Sobajima J, Kumagai Y, Ishibashi K, Ishida H. Prognostic Factors for Gastric Cancer with Cancer Cells in the Peritoneal Cavity. Anticancer research. 2016; 36:2481-2485.

37. Sadeghi B, Arvieux C, Glehen O, Beaujard AC, Rivoire M, Baulieux J, Fontaumard E, Brachet A, Caillot JL, Faure JL, Porcheron J, Peix JL, Francois Y, et al. Peritoneal carcinomatosis from non-gynecologic malignancies: results of the EVOCAPE 1 multicentric prospective study. Cancer. 2000; 88:358-363.

38. Yoo CH, Noh SH, Shin DW, Choi SH, Min JS. Recurrence following curative resection for gastric carcinoma. The British journal of surgery. 2000; 87:236-242.

39. Moriguchi S, Maehara Y, Korenaga D, Sugimachi K, Nose Y. Risk factors which predict pattern of recurrence after curative surgery for patients with advanced gastric cancer. Surgical oncology. 1992; 1:341-346.

40. Kobayashi H, Kotake K, Sugihara K. Prognostic significance of peritoneal lavage cytology in patients with colorectal cancer. International journal of clinical oncology. 2013; 18:411-417.

41. Gaspar MJ, Arribas I, Coca MC, Diez-Alonso M. Prognostic value of carcinoembryonic antigen, CA 19-9 and CA 72-4 in gastric carcinoma. Tumour biology. 2001; 22:318-322.

42. Kwon OK, Yu W, Chung H. Prognostic value of postoperative CA19-9 normalization in patients with advanced gastric cancer. Hepato-gastroenterology. 2013; 60:240-243.

43. Kochi M, Fujii M, Kanamori N, Kaiga T, Kawakami T, Aizaki K, Kasahara M, Mochizuki F, Kasakura Y, Yamagata M. Evaluation of serum CEA and CA19-9 levels as prognostic factors in patients with gastric cancer. Gastric cancer. 2000; 3:177-186.

44. Lorenzen S, Panzram B, Rosenberg R, Nekarda H, Becker K, Schenk U, Hofler H, Siewert JR, Jager D, Ott $\mathrm{K}$. Prognostic significance of free peritoneal tumor cells in the peritoneal cavity before and after neoadjuvant chemotherapy in patients with gastric carcinoma undergoing potentially curative resection. Annals of surgical oncology. 2010; 17:2733-2739.

45. Cotte E, Peyrat P, Piaton E, Chapuis F, Rivoire M, Glehen O, Arvieux C, Mabrut JY, Chipponi J, Gilly FN. Lack of prognostic significance of conventional peritoneal cytology in colorectal and gastric cancers: results of EVOCAPE 2 multicentre prospective study. European journal of surgical oncology. 2013; 39:707-714.

46. Yamamoto M, Baba H, Kakeji Y, Endo K, Ikeda Y, Toh Y, Kohnoe S, Okamura T, Maehara Y. Prognostic significance of tumor markers in peritoneal lavage in advanced gastric cancer. Oncology. 2004; 67:19-26. 
47. Kanetaka K, Ito S, Susumu S, Yoneda A, Fujita F, Takatsuki M, Kuroki T, Eguchi S. Clinical significance of carcinoembryonic antigen in peritoneal lavage from patients with gastric cancer. Surgery. 2013; 154:563-572.

48. Hasegawa H, Fujitani K, Nakazuru S, Hirao M, Yamamoto K, Mita E, Tsujinaka T. Optimal treatment change criteria for advanced gastric cancer with non-measurable peritoneal metastasis: symptom/tumor marker-based versus CT-based. Anticancer research. 2014; 34:5169-5174. 